\title{
A Neurobiological Model for Near-Death Experiences. II: The Problem of Recall of Real Events
}

\author{
Juan Sebastian Gómez-Jeria, Lic. Q. \\ Juan Carlos Saavedra-Aguilar, M.D. \\ University of Chile
}

ABSTRACT: In this article we propose a scientific approach to explain the fact that some near-death experiencers (NDErs) are able to recollect and verbalize real events occurring in the environment during the experience. Our model assigns a central place to priming, multiple declarative memory, and verbal modules. These biological mechanisms lead to the assimilation of multiple external cues, the consolidation in memory of matched primed environmental events, and the transformation and creation of logically structured functional engrams. Finally, the after-NDE behavioral and verbal interactions between the experiencer and a community of observers are discussed, together with their results.

Until now, near-death studies have been carried out along several approaches, some of which are incompatible. The emergence of neurobiological models strongly suggests that near-death studies have reached a stage allowing a systematic and rigorous organiza-

Juan Sebastian Gómez-Jeria, Lic.Q., is Associate Professor in the Faculty of Sciences, and Juan Saavedra-Aguilar, M.D., is Assistant Professor in the Faculty of Medicine, both at the University of Chile. This work was funded by research grants from the International Association for Near-Death Studies and from the University of Chile (DTI Project Q-3064). The opinions within this article are the sole responsibility of the authors. Dr. Bruce K. Cassels is gratefully acknowledged for his kind help and comments. Reprint requests should be addressed to Lic. Gómez-Jeria at the Universidad de Chile, Facultad de Ciencias, Departamento de Quimica, Casilla 653, Santiago, Chile. 
tion. Naturally, the task of squeezing a large body of facts into a model can be a painful one, especially when personal beliefs are involved. The presence of the really arbitrary, such as religious and personal beliefs, and the moderately arbitrary, which are necessary in the first steps in the construction of a scientific model, results in the existence of various basically different "explanations" for the same phenomenon.

In the case of near-death experiences (NDEs), a detailed neurobiological model has been proposed (Saavedra-Aguilar and GómezJeria, 1989a, 1989b). Later, some extensions or variations of it were presented (Appleby, 1989; Jansen, 1989a, 1989b, 1990; Morse, Venecia, and Milstein, 1989; Owens, Cook, and Stevenson, 1990). Some of these authors seemed to be unaware of the near-death research literature. Of course, the proprietary questions are not of much importance, but it is strictly necessary to keep the literature straight. A short time ago, an approach we feel was methodologically erroneous was used to find similarities between some NDEs and multiple personality disorder (MPD) (Serdahely, 1992). Considering that is has been shown that there is a phenomenological overlap between MPD and some cases of obsessive-compulsive disorder (Ross and Anderson, 1988), we might conclude erroneously that perhaps some NDEs have similarities with some obsessive-compulsive disorders. Naturally, this is not the case: the NDE is not a clinical disorder.

In addition there have been proposed nonscientific models such as the "transcendental" one, in which personal beliefs are mixed with religious traditions (Basil, 1991). We believe that time will show that scientific models are the only ones accounting for neardeath phenomena. In this article, we will center our work in scientific terms. A tentative working strategy for developing this model is shown in Figure 1, with the understanding that scientific models always need to be ameliorated in the light of new knowledge (Nagel, 1991).

The problem that will be explored here is the following: some persons are able to recollect and verbalize, at least partially, real events, such as a nurse's voice or the color of a doctor's clothing, occurring in the environment during an NDE. We shall present and discuss recent evidence about the functional mechanisms by which the nervous system of a person in an abnormal state is able to deal with the environment. This article initiates a scientific approach to the abovementioned aspect of the NDE. 
Figure 1

A Simplified Strategy for a Scientific Approach to NDEs

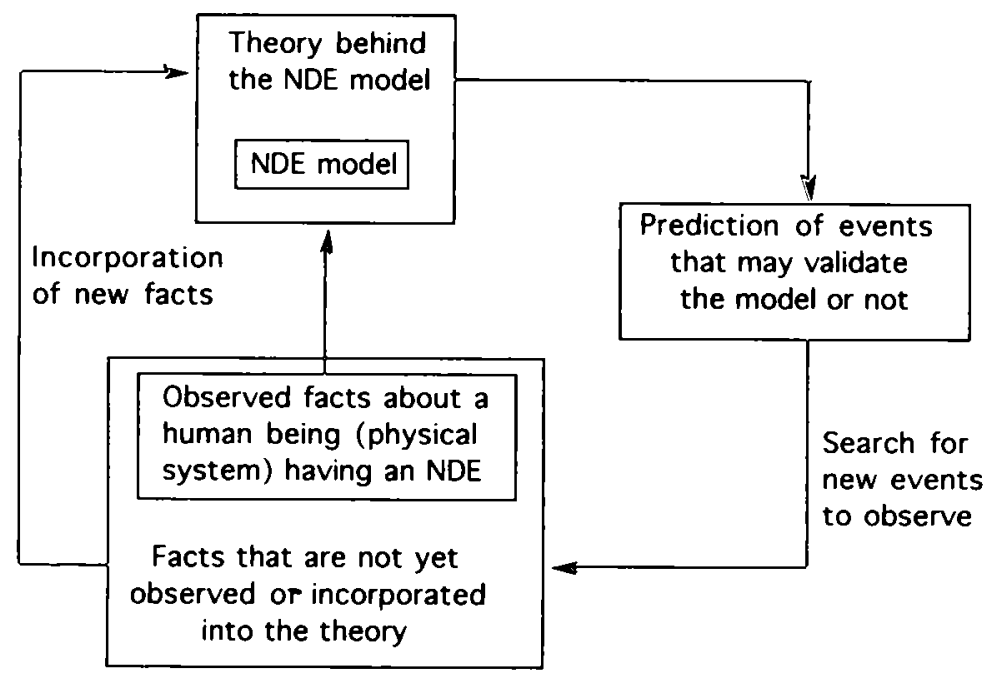

\section{Neurobiological Evidence and the Model}

\section{Anesthesia and Environmental Awareness}

During anesthesia, 1 to 75 percent of patients show variable states of awareness of the environment, but with no recollection of it upon awakening. Furthermore, conscious recall of contextual environmental events during a surgical procedure is reported by about 1 percent of patients (Kulli and Koch, 1991; Schultetus, Hill, Dharamraj, Banner, and Berman, 1986). In recall, the patient remembers and communicates intraoperative events during the surgical procedure. More strikingly, priming effects have been reported in anesthetized subjects, although the existence of such effects has been challenged by some reports (Eich, Reeves, and Katz, 1985; Kulli and Koch, 1991; Levinson, 1965; Maintzer, 1979).

The evidence indicates that, despite the anesthetized organism being in a state hindering reaction to the environment, the environment can influence the organism, which may then behave in a more or less appropriate way. It is therefore arguable that, during the NDE, some persons may use the same biological mechanisms underlying 
awareness and recall during anesthesia. These mechanisms seem to involve the various processes sustaining learning and the memory of events taking place in the environment. Furthermore, for the subsequent conscious communication of these processes, the verbal functional system seems essential.

\section{Learning and Memory Processes: The Importance of Priming}

If an organism is able to adapt to the environment, it has to learn (that is, consolidate in some functional way in its structure) contingent aspects of the environment. This process allows the subsequent reconstruction of this particular configuration with regard to different sets of relational environmental events, generating the memory processes and their behavioral consequences. Memory processes have been classified as unconscious procedural memories and conscious verbal declarative memories (Baddeley, 1987; Squire, 1987). A basic mechanism that seems to underlie procedural and declarative memory appears to correspond to priming.

Priming refers to the implicit facilitation of sensory events and their behavioral results, brought about by widely different unconscious and subliminal environmental stimuli, which nevertheless can be structured as sets of specific functional processes in early neural sensorimotor processing (Squire, 1992; Tulving and Schacter, 1990). In a classic experimental paradigm, different stimuli, such as letters or geometric figures, are tachistoscopically presented to the subject during brief intervals of 1-2 milliseconds. The subject does not report any conscious recall of these stimuli. Later, when a list of related and unrelated stimuli are presented and the subject is instructed to choose some of them, the contingent stimuli are chosen more frequently than the unrelated ones. Priming appears to involve the sensorimotor primary areas; letters and forms appear to be structures in right posterior brain regions, while sequences of words are primed in the left posterior brain regions (Squire, 1992).

Priming is affected by the environmental context and by the affective state of the subject (Bornstein and Pittman, 1992; Niedenthal, 1992). It is a primary mechanism that can be used by long-term declarative memory processes and by short-term memory processes (Squire, 1987), and it is also essential for procedural learning. The affective state of the organism could modulate the priming mechanism through the connections between limbic structures, such as the 
amygdala, and the primary sensory areas. Since these regions are important in declarative memory, the content of these memories can affect priming. The procedural memories could act on priming in sensorimotor areas through the connections between the basal ganglia, including thalamic nuclei, and these regions. We suggest here that the effect of declarative and nondeclarative memories on priming seems to provide the fine tuning of this process to more restricted environmental aspects. On the contrary, it is expected that in cases of decreased alertness, such as during anesthesia and near-death situations, this abnormal state results in a priming mechanism functioning in a less restricted way.

The net result of the latter situation would permit priming to be gated by a wider set of environmental events. In this biological condition, declarative memory processes could access a broader set of primed representations of the environment. This would result in the possibility of "knowing," by using declarative memory and its necessary related verbal mechanism, different actual events that occur during, for example, a surgical procedure. In the case of NDEs, the same mechanism could permit the recall of wider aspects of the environmental situation related to this experience. To understand fully the implication of this possibility, it is useful to discuss some aspects of verbal mechanisms and their relevance to this situation.

\section{The Verbal System and Its Relevance in the Unification of Consciousness}

Michael Gazzaniga (1985) discussed the experimental evidence regarding the role of the verbal system in making "logical" verbal inferences dealing with different behaviors and affective states of a subject, and its role in the generation of the self. Based on evidence such as split-brain patients, he suggested that different modules of the brain permanently elicit different nonverbal behaviors and affective states. A specialized system, the verbal behavioral system, generates different beliefs related to each situation. This process is essential for making sense of various behavioral repertoires and affective states.

This operation is based on past sociocultural and individual experiences and integrates these different elements into a more or less coherent and socially plausible hypothesis, resulting in a personal belief system (Saavedra-Aguilar and Gómez-Jeria, 1991). This mecha- 
nism uses declarative memory processes that, in turn, are modulated recursively by different processes such as priming. As we said above, in abnormal states, such as those associated with anxiety or placidity, the declarative memory processes could gain access to a wider set of environmentally-primed events. In these situations, the verbal system is flooded with declarative neural processes that, in turn, are based on this wide priming structuring occurring in primary areas.

As postulated by Gazzaniga (1985), this jigsaw puzzle of environmental events can be integrated by this system through the generation of a coherent personal hypothesis, or belief. Hence, this belief can be integrated with previous knowledge, resulting in a coherent story. This "story," corresponding to a specific belief about a situation, can be highly accurate, compared to the actual events that gated the priming processes. As the verbal system functions in a logically structured way, the subject knows that he or she was paralyzed, with eyes closed, but nevertheless heard the doctor's voice and the noises of the surgical suite. A good "hypothesis" is to create the belief that he or she was looking at this situation from above, by using and gating the access of previous contextual visuokinesthetic memories, such as the ones that are common in some dreams.

These memory fragments are integrated with the primed related declarative memories, resulting in the belief that the subject was actually observing the situation, that is, having an out-of-body experience (OBE). Since this can generate accurate reports about the actual conditions of the situation, accessed through the facilitated primed mechanisms, the belief is strengthened and consolidated in the subject's personal biography and in the beliefs of the audience. It should be clearly understood that this mechanism does not operate only in verbal terms, but uses recursively different visuokinesthetic memory processes and memories of personal beliefs, such as religious ones. Nevertheless, this specific explanation for some OBEs does not exclude other mechanisms to explain this mental experience.

\section{Commentary}

The model suggested above integrates the personal history and beliefs of a subject with neural mechanisms representing the actual events that took place during the episode. This model is summarized in Figure 2. Since some data of the experiencer's story can be corroborated by another observer or by a community of observers, this 


\section{Figure 2 \\ The Model for Recall of Real Events Occurring During an NDE}

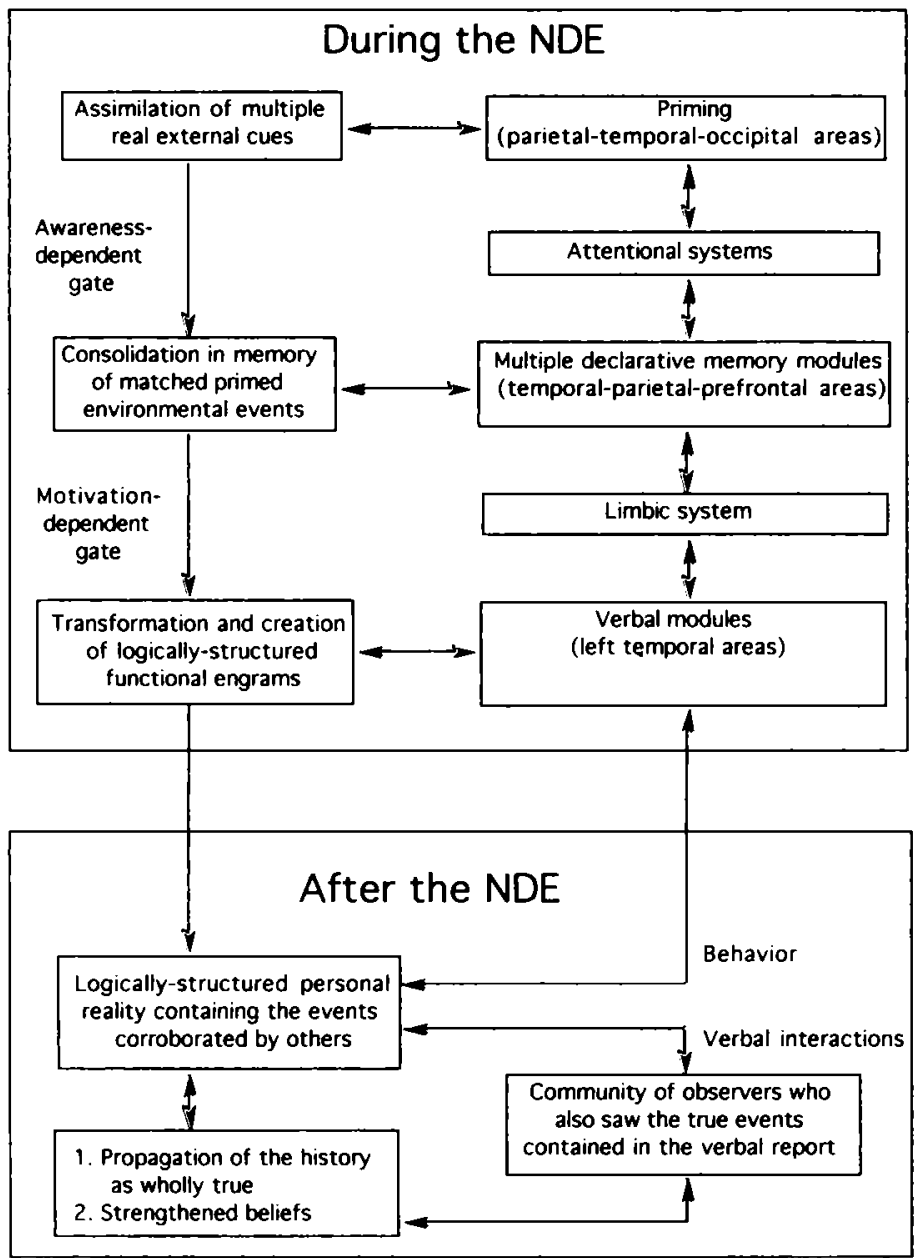

leads other people to believe the story - which, by a subsequent feedback mechanism, reinforces the subject's belief. Because most neardeath experiencers are not really near death (Gabbard and Twemlow, 1991; Gabbard, Twemlow, and Jones, 1981; Owens, Cook, and Stevenson, 1990), and because in the verbal report of the story there appear 
some elements of the subject's own culture (Gómez-Jeria, 1993), we get the propagation of an "acceptable" tale. Given that this tale demands an explanation as a complete truth, the final stage of this process is the production of several sets of nonscientific statements claiming to be "explanations."

We must add that the attempt to redefine the NDE as an experience occurring only during a real near-death situation is a groundless way of trying to keep NDEs from being explained. We hope that this paper will open a serious discussion about the nature of the different stages of the near-death experience and their contents, taken as, for example, elements appearing in the field of consciousness.

Finally, it would be desirable to discuss the advisability of changing the name of the NDE. A change of name will not modify the fascinating nature of this mental experience, but it will change several prejudices about its nature. We strongly feel that the vocabulary actually in use is an obstacle that the theory has to overcome, just as the search for a theory is hindered by the constraints of some habits of thought regarding astral planes and souls.

\section{References}

Appleby, L. (1989). Near-death experience: Analogous to other stress induced psychological phenomena. British Medical Journal, 298, 976-977.

Baddeley, A. (1987). Working memory. Oxford, England: Clarendon.

Bornstein, R. F., and Pittman, T. S. (Eds.). (1992). Perception without awareness. New York, NY: Guilford

Eich, E., Reeves, J. L., and Katz, R. L. (1985). Anesthesia, amnesia and the memory/awareness distinction. Anesthesia and Analgesia, 64, 1143-1148.

Gabbard, G. O., and Twemlow, S. W. (1991). Do "near-death experiences" occur only near death? - Revisited. Journal of Near-Death Studies, 10, 41-47.

Gabbard, G. O., Twemlow, S. W., and Jones, F. C. (1981). Do "near-death experiences" occur only near death? Journal of Nervous and Mental Disease, 169, 374-377.

Gazzaniga, M. S. (1985). The social brain. New York, NY: Basic Books.

Gómez-Jeria, J. S. (1993). A near-death experience among the Mapuche people. Journal of Near-Death Studies, 11, 219-222.

Jansen, K. L. R. (1989a). Near-death experience and the NMDA receptor [Letter]. British Medical Journal, 298, 1708.

Jansen, K. L. R. (1989b). The near-death experience [Letter]. British Journal of Psychiatry, 154, 883-884.

Jansen, K. L. R. (1990). Neuroscience and the near-death experience: Roles for the NMDA-PCP receptor, the sigma receptor and the endopsychosins. Medical Hypotheses, 31, 25-29.

Kulli, J., and Koch, C. (1991). Does anesthesia cause loss of consciousness? Trends in Neurosciences, $14,6-10$.

Levinson, B. W. (1965). States of awareness during general anaesthesia. British Journal of Anaesthesia, 37, 544-546. 
Maintzer, J., Jr. (1979). Awareness, muscle relaxants and balanced anaesthesia. $\mathrm{Ca}$ nadian Anaesthetists' Society Journal, 26, 386-392.

Morse, M. L., Venecia, D., Jr., and Milstein, J. (1989). Near-death experiences: A neurophysiological explanatory model. Journal of Near-Death Studies, 8, 45-53.

Nagel, E. (1991). La estructura de la ciencia. Barcelona, Spain: Ediciones Paidos.

Niedenthal, P. M. (1992). Affect and social perception: On the psychological validity of rose-colored glasses. In Bornstein, R. F., and Pittman, T. S. (Eds.), Perception without awareness. New York, NY: Guilford.

Owens, J. E., Cook, E. W., and Stevenson, I. (1990). Features of "near-death experience" in relation to whether or not patients were near death. Lancet, 336, 1175-1177.

Ross, C. A, and Anderson, G. (1988). Phenomenological overlap of multiple personality disorder and obsessive-compulsive disorder. Journal of Nervous and Mental Disease, 176, 295-299.

Saavedra-Aguilar, J. C., and Gómez-Jeria, J. S. (1989a). A neurobiological model for near-death experiences. Journal of Near-Death Studies, 7, 205-222.

Saavedra-Aguilar, J. C., and Gómez-Jeria, J. S. (1989b). Response to commentaries on "A neurobiological model for near-death experiences." Journal of Near-Death Studies, 7, 265-272.

Saavedra-Aguilar, J. C., and Gómez-Jeria, J. S. (1991). On the origin and neural organization of intentionality: A theoretical proposal. Revista Chilena de NeuroPsiquiatria, 29, 187-198.

Schultetus, R. R., Hill, C. R., Dharamraj, C. M., Banner, T. E., and Berman, L. S. (1986). Wakefulness during cesarian section after anesthetic induction with ketamine, thiopental, or ketamine and thiopental combined. Anesthesia and Analgesia, 63, 723-728.

Serdahely, W. J. (1992). Similarities between near-death experiences and multiple personality disorder. Journal of Near-Death Studies, 11, 19-38.

Squire, L. R. (1987). Memory and brain. New York, NY: Oxford University Press.

Squire, L. R. (1992). Memory and the hippocampus: A synthesis from findings with rats, monkeys and humans. Psychological Review, 99, 195-231.

Tulving, E., and Schacter, D. L. (1990). Priming and human memory systems. Science, 247, 301-306. 